\title{
Swing Node on State Estimation Results with Bad Data and its Error Analysis
}

\author{
Jian Guo ${ }^{1,}$, , Yin Zhang ${ }^{1}$, Yihang Chen ${ }^{2,}$, , Ruzhi $\mathrm{Xu}^{2}$ \\ ${ }^{1}$ Power System Automation Department China Electric Power Research Institute Beijing, China \\ ${ }^{2}$ School of Control and Computer Engineering North China Electric Power University Beijing, China \\ aguojian_715@126.com, b20002810@qq.com
}

Keywords: Swing node, state estimation, iteration correction, bad data, error analysis.

Abstract. Swing node is the basis of computation for state estimation, to analysis its impact on state estimation, especially when there is bad data around swing node, this paper did detailed analysis and case studies on whether swing node involved in iteration computation or not. There are three aspects of cases, which are swing node with bad measurement, bad parameter and adjacent nodes having bad data etc. IEEE-14 was used for simulation and data analysis; experiment error analysis results show that in case of swing node not being involved into the iterations can obtain better estimate results.

\section{Introduction}

In power system steady and on-line analysis calculations, swing node generally used as the reference node, and takes the responsibility of power balance of the final results. It is important to select a swing node, not only for power flow computation, but also for state estimation. There are a lot of literatures about the impact of swing selection on power flow calculation. In state estimation area, it is always possible to have bad measurement data, which has an important influence on accuracy and precision, so bad data detection and identification have got a lot of attentions. But thoroughly investigation on how and how much influences swing node with bad data impact on final results, there is little literature published.

In theory, as long as the number of equations constructed are linearly independent greater than number of unknown arguments, then the equations can be solved base on LSE, estimation values such as node voltage variables, network parameter only have to satisfy convergence criterion. The following chapter will focus on effect of final result about whether voltage amplitude of swing node involves into iterations correction or not, and compare results in quantitative when bad data and parameters exist around swing node.

\section{Mathematical Theory}

The measurement equation of state estimation is as follows:

$\mathrm{z}=\mathrm{h}(\mathrm{x})+\mathrm{v}$

Where:

$\mathrm{x}$ is node phasor,

$\mathrm{n}$ is number of the nodes,

$\mathrm{z}$ is measurement vector,

$m$ is the number of measurement $(m>n)$,

$\mathrm{h}$ is nonlinear equations on nodes phasor,

$\mathrm{v}$ is measurement error, it is generally assumed to meet $(0, \sigma 2)$ normal distribution.

Weighted least square iterative correction formula is:

$\mathrm{G}_{\mathrm{k}} \Delta \mathrm{x}_{\mathrm{k}}=\mathrm{H}_{\mathrm{k}}^{\mathrm{T}} \mathrm{W}\left[\mathrm{z}-\mathrm{h}\left(\mathrm{x}_{\mathrm{k}}\right)\right]$

Where:

$\mathrm{H}_{\mathrm{k}}=\partial \mathrm{h} / \partial \mathrm{x}$ is Jacobi matrix which calculated in first-order partial derivatives,

$\mathrm{G}_{\mathrm{k}}=\mathrm{H}_{\mathrm{k}}^{\mathrm{T}} \mathrm{WH}_{\mathrm{k}}$ is gain matrix,

$W=\operatorname{diag}\left(W_{i}\right)$ is weighted diagonal matrix, generally the reciprocal of covariance of measurement error $\sigma_{i}^{2}$ as weight vector $W_{1}$. 
$\Delta \mathrm{x}_{\mathrm{k}}=\mathrm{x}_{\mathrm{k}+1}-\mathrm{x}_{\mathrm{k}}, \mathrm{k}$ is number of iterations. The iteration stops when $\Delta \mathrm{x}_{\mathrm{k}}$ satisfy convergence criterion.

The dimension of Jacobian matrix $\mathrm{H}$ is $2(\mathrm{n}-1)$ or $2 \mathrm{n}-1$ depends on whether swing node involves into iterative correction, if it is $2 \mathrm{n}-1$, the added column vector forms as (3):

$\frac{\partial \mathrm{h}}{\partial v}=\left[\frac{\partial \mathrm{V}}{\partial \mathrm{v}}, \frac{\partial \mathrm{p}}{\partial \mathrm{v}}, \frac{\partial \mathrm{Q}}{\partial \mathrm{v}}, \frac{\partial \mathrm{P}_{\mathrm{if}}}{\partial \mathrm{v}}, \frac{\partial \mathrm{Q}_{\mathrm{il}}}{\partial \mathrm{v}}\right]^{\mathbf{T}}$

$\partial \mathrm{V} / \partial \mathrm{v}$ first-order partial derivative of node voltage amplitude to swing amplitude,

$\partial \mathrm{P} / \partial \mathrm{v}, \partial \mathrm{Q} / \partial \mathrm{v}$ first-order partial derivative of node active and reactive injected power to swing node amplitude,

$\frac{\partial \mathrm{P}_{\mathrm{ij}}}{\partial \mathrm{v}}, \frac{\partial \mathrm{Q}_{\mathrm{ij}}}{\partial \mathrm{v}}$ First-order partial derivative of branch ends active and reactive power flow to swing node amplitude.

Due to swing node amplitude involves into iterative correction, its value changes during each iteration, so the branches power flow connected with swing node also change. In addition, corresponding to swing node without involving into iterative correction, it will bring different correction values of $\Delta \mathrm{x}_{\mathrm{k}}$, which affect the final result. Detailed explanations need to be expanded in next chapters.

\section{Data Preparation and Process}

IEEE-14 node data is modified for testing, which measurements are added on bus node and line, figure1 shows its diagram.

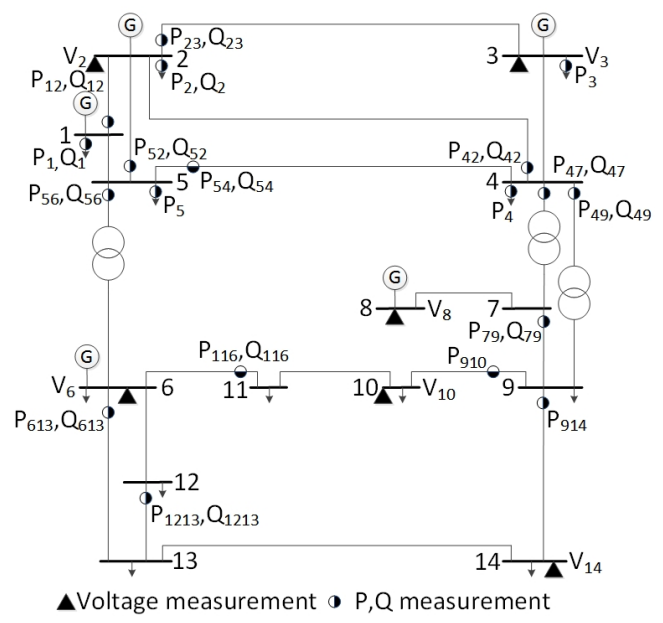

Figure 1. IEEE 14-node with measurements

Power flow data is used as basis and reference data, while Gaussian noisy is added to simulate measurement data, a simple observability analysis is:

(1) Number of voltage measurement spots is 6 , greater than 1 ;

(2)Number of active power measurement spots is 19 , greater than 13 ;

(3) Number of reactive and voltage measurement spots is 20 , greater than 14 ;

According to the above 3 criterions, measurement spots configuration meet observability requirements of system. Choose bus 2 as swing node and the calculation mode is plat start. Computation and comparison process shows in figure2.

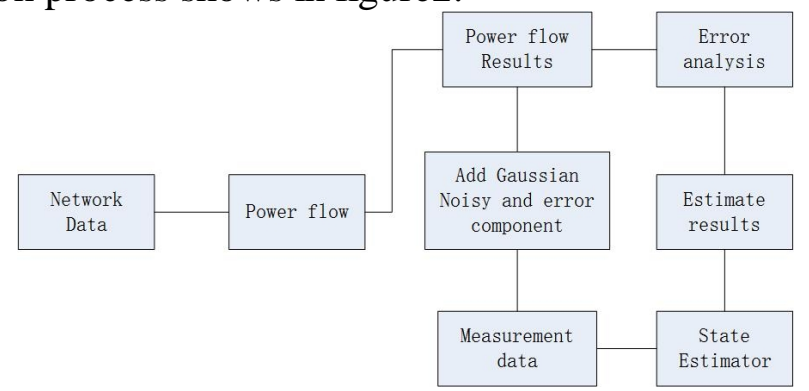

Figure 2. Error analysis process 


\section{Results Comparison with Normal Data}

To compare the results on whether swing node involved into iteration correction or not, in condition of network parameters and measurement devices are normal, both have to converge in 6 time iterations. Error comparison between node voltage amplitude and phase angle and corresponding real value of power flow calculation shows in figure 3.

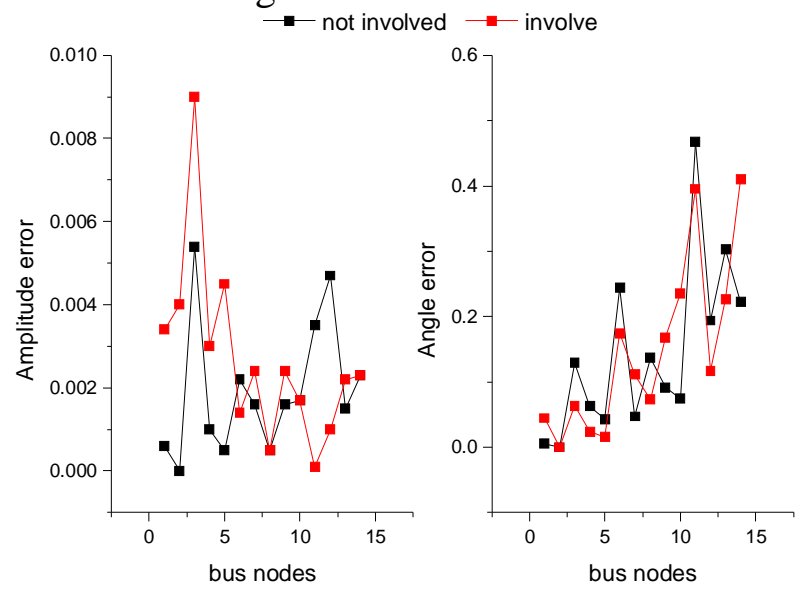

Figure 3. Error analysis on whethter swing node involved or not with normal data

The figure shows that, when swing node not involves into iterative corrections, there is smaller difference between estimated and true values, but when swing node involves into iterative corrections, caused amplitude fluctuation within few thousandths, and phasor amplitude fluctuation within few percent. So in condition of network parameters and measurement devices are normal, whether swing node participates in iterative calculation or not has little impact on the final results.

Since transmission lines usually transmit electric in double or more back, one of the lines inevitably breaks down or occurs short-circuit fault, which changes line parameters, while to maintain new transmission line model and parameters need longer maintenance cycle. So there exists parameter inconsistency between real physical parameters and calculation parameters. To simulate the situation, impedance parameter of line 1-2 which closes to swing node is amplified 2-fold and narrowed to 1/2 one time, while the real network parameters still unchanged. Swing node involves into iteration correction or not is separated to compare with the true value calculated by power flow shows in Figure 4.

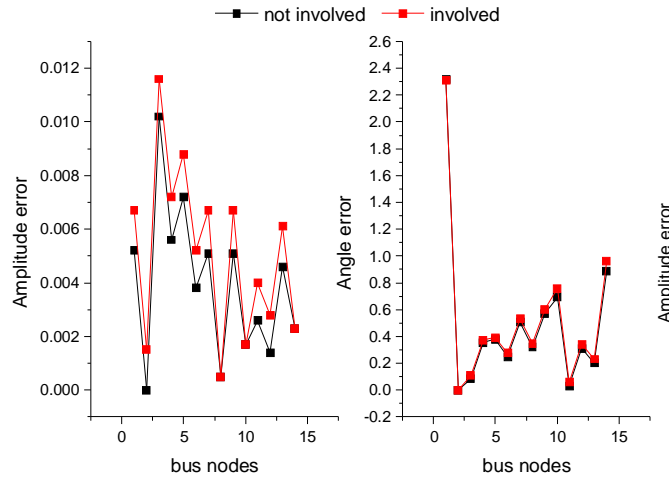

(1) Amplified to 2 times

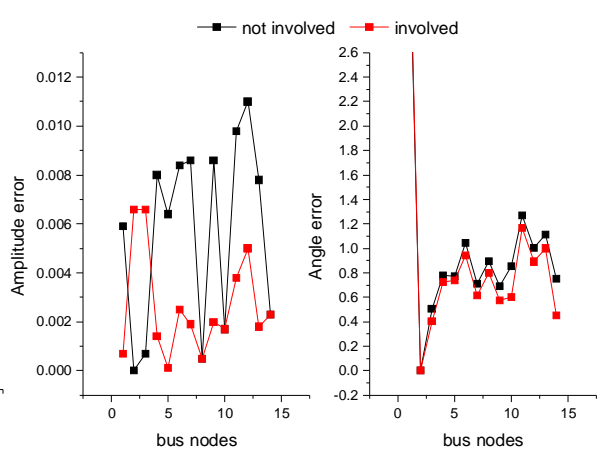

(2) Reduced to $1 / 2$

Figure 4. Error analysis on whethter swing node involved or not with line parameter changed

When the parameters of the branch model near the slack bus are not updated in time, or when the failure occurs, whether the slack bus is involved in the iterative calculation has little effect on the estimation results. When the branch parameter increases to 2 times, the errors of bus magnitude and phase angle are basically synchronous. When the branch parameter is reduced to $1 / 2$, the bus magnitude has greater volatility, the error caused by the calculation involving slack bus is small, and the variation of phase angle error always stay in sync. 


\section{Results Comparison with Bad Data}

\section{A.The magnitude of the swing bus as bad data}

The error components of $10 \%, 20 \%, 30 \%$ and $50 \%$, are added to the voltage measurement magnitude of the swing bus 2 . Respectively, analyzed and compare the both cases, the swing bus participating in iterative correction and is not involved in the iterative correction, see figure5.

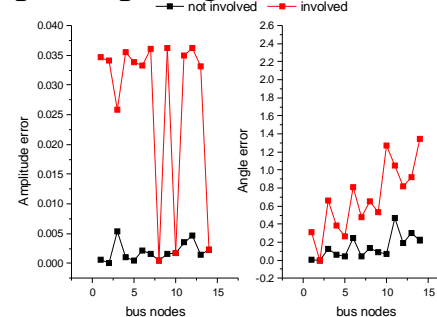

(1) $10 \%$

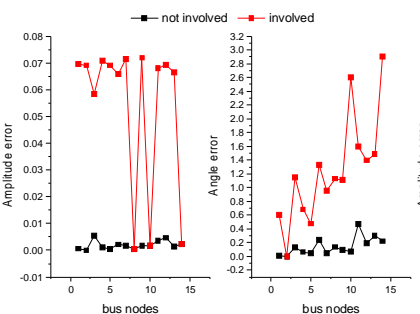

(2) $20 \%$
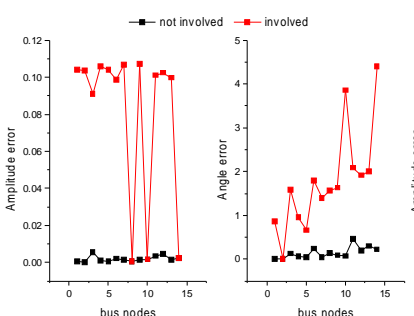

(3) $30 \%$

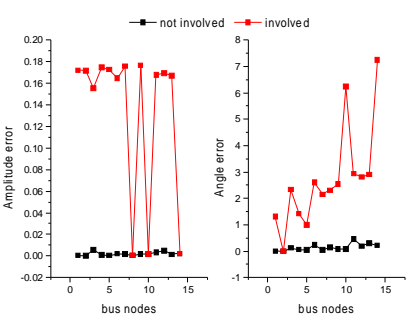

(4) $50 \%$

Figure 5. Error analysis with 10\%, 20\%, 30\%, 50\% error component in swing bus amplitude

The presence of bad data measuring swing bus, whether swing bus involve in the Iterative correction or not will result in a larger calculation error. It can be seen from the diagram, swing bus is involved in the iterative correction compared with is not involved in the iterative correction, except $8,10,14$ bus, the remaining bus voltage amplitude have larger error, bus phase error of the bus with the swing bus electrical distance increases, showing a growing trend. Generally speaking, with the measurement error component of the swing bus growing, estimated the bus magnitude and phase angle difference between the true values is large. When the error component is greater than $30 \%$, the state estimator has lost its meaning. Thus, when the swing bus involved in iteration correction, it is need to ensure the accuracy of the amplitude measurement.

\section{B. Branch measurement adjacent to swing bus as bad data}

The error components of 10\%, 20\%, 30\% and 50\%, are added to the Branch flow measurement P123 adjacent to the swing bus. Respectively, analyzed and compare the both cases, the swing bus participating in iterative correction and is not involved in the iterative correction, see figure 6 .

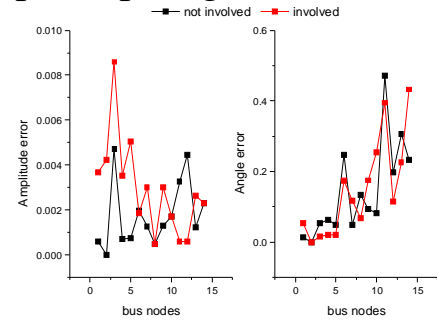

(1) $10 \%$

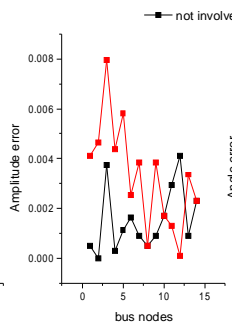

(2) $20 \%$

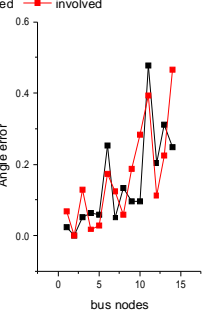

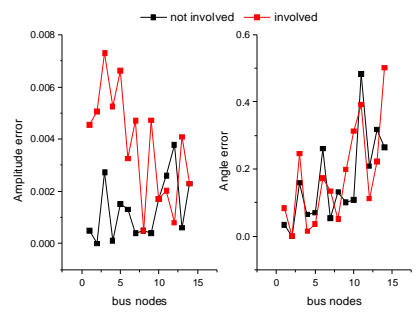

(3) $30 \%$

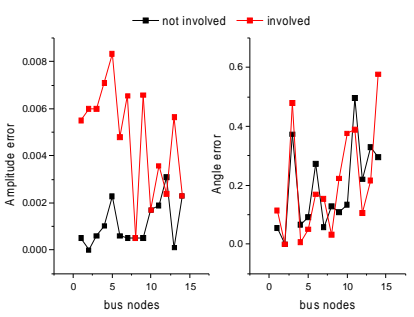

(4) $50 \%$

Figure 6. Error analysis with 10\%, 20\%, 30\%, 50\% error component in line measurements adjcent to swing bus

From figure6, we can see that when swing node is measured normally while adjacent line 2-3 has measurement errors, if swing node involves into iteration correction, the nodes voltage amplitude error which are connected to the line is bigger. With respect to non-participation into the iterative correction mode, voltage amplitude of each node fluctuates more. Compared to the measurement error existence in swing node, the amplitude and phasor error of each node fluctuates little with line measurement error increased. Overall, if swing node not involved into the iterative correction, estimation results will be better.

\section{C.Node voltage measurement adjacent to swing node as bad data}

The error components of $10 \%, 20 \%, 30 \%$ and $50 \%$, are added to the voltage amplitude measurement Bus3 adjacent to the swing bus. Respectively, analyzed and compare the both cases, the swing bus participating in iterative correction and is not involved in the iterative correction, see figure 7. 


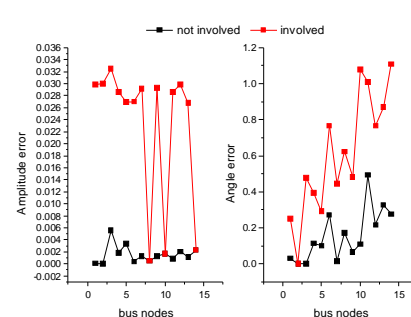

(1) $10 \%$

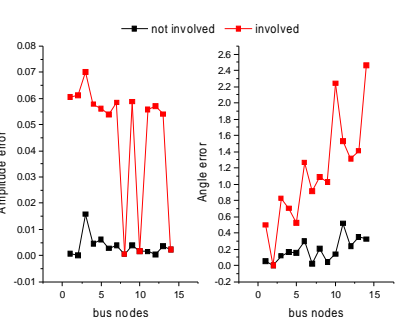

(2) $20 \%$

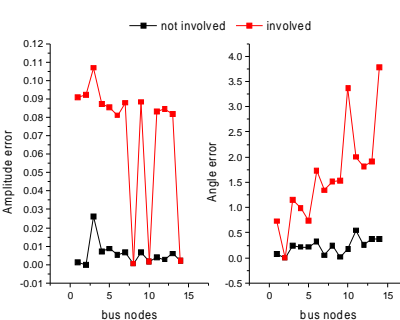

(3) $30 \%$

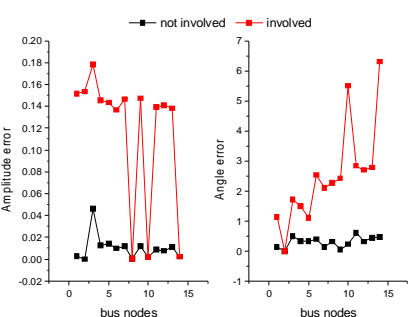

(4) $50 \%$

Figure 7. Error analysis with $10 \%, 20 \%, 30 \%, 50 \%$ error component in bus amplitude adjcent to swing bus

Similar to the situation about swing node joins deviation component, in the position of the swing node involvement iterative correction, the node 3 have the maximum estimated deviation and as the same tendency to the nodes $1,2,4$ which is directly connected to the node 3 . With the increase of measurements deviation component, the node estimated deviation value is also growing, and the node phase angle deviation present increasing trend.

\section{Summary}

The experiments above showed that, it can get better state estimation result that swing node voltage amplitude not involving in the iterative correction when accuracy of the measurement of the amount of balance node can't be guaranteed. Temporary changes in model parameters of power system have less affect in state estimation calculation results; meanwhile if the measurements of branch and voltage adjacent to the swing node have a more deviation component, there will bring the estimated value of voltage and phase angle near the swing node more deviation, causing residual contamination. Therefore, the research on how to minimize the pollution range of measurement bad data is a necessity.

\section{Acknowledgements}

This work was financially supported by the Science and Technology Project of State Grid (Project No. DZ71-14-040).

\section{References}

[1] Heyang zan, Wenzengyin.Power system analysis[M]. HuaZhong University of Science and Technology Press, 2001.

[2] Miao Fengxian , Bai Xuefeng, Guo Zhizhong. A Power Flow Algorithm Considering Reactive Power Constraints of Slack Bus[J] . Power System Technology , 33(9) : 52-56 , 2009

[3] Zhang Chao, Wu Zhengqiu , Zhong Hao , Li Ribo[J] . Proceedings of the CSUEPSA , 23(6) : $127-130,2011$.

[4] Ding Hongen , Gao Zonghe ,Su Dawei, et al . Solution to Bad Problem of PhaseAngle Reference Bus for State Estimation with Hybrid Measurement[J] .Automation of Electric Power Systems .38(9) : $132-136,2014$

[5] Li Qiang , Zhou Jingyang, YU Erkeng, A Hybid Algorithm for Power System State Estimation Based on PMU Measurement and SCADA Measurement[J] . Automation of Electric Power Systems , 29(19) : 31-35, 2005.

[6] F C.SCHWEPPE. "Power System Static-State Estimation, Part I, II, III,"IEEE Trans.Power Syst., vol.PAS-89,no.1,pp.120-135,Jan.1970. 
[7] Guo Ye, Zhang Boming, Wu Wenchuan . Solution and Performance Analysis to a Robust State Estimation Method With Exponential Objective Function[J] .Proceedings of the CSEE ,31(7): :89-95 , 2011 . 